\title{
Design of the Spherical Interface Between the CC Stanchions and Carriage
}

Engineering Note \#3740.214-EN-103

Issued: August 7, 1987

Revised: September 25, 1987

Originator: Richard D. Luther 
loured 9/23/87

DETERMINE THE RADIUS OF THE SAMEPICAC INTERACT BETWEEN THE STANCHION AND THE CARnAGE ON THE ce cryostat.

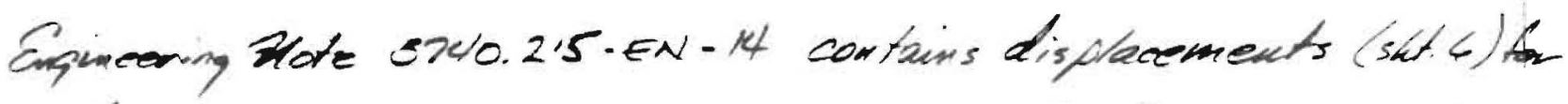
The support plate on the carriage under tho hell weight of the ce calorimeter, determined by Finite thereat Analysis (FEA). These are:

(Sente) $<$

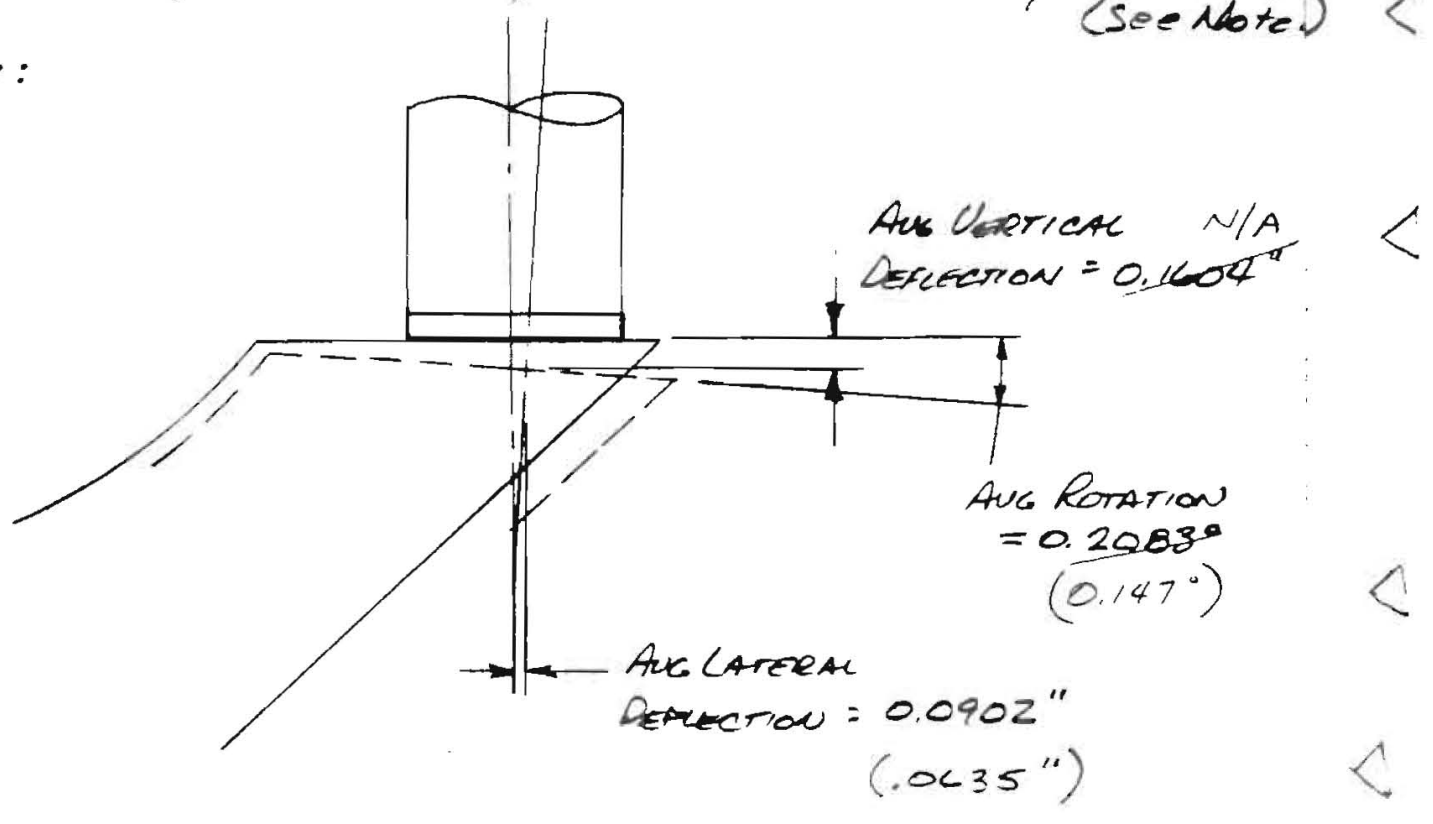

The spherical inter tace is sized to ensure that tie vertical countermine of the stanciven does not move laterally as the carriage deflects.

Note: Number in parentheses about are taken Flem

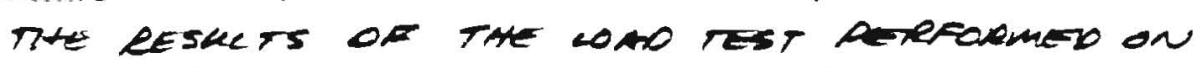

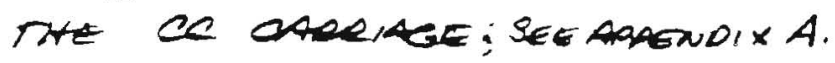


exe $8 / 7 / 87$

2

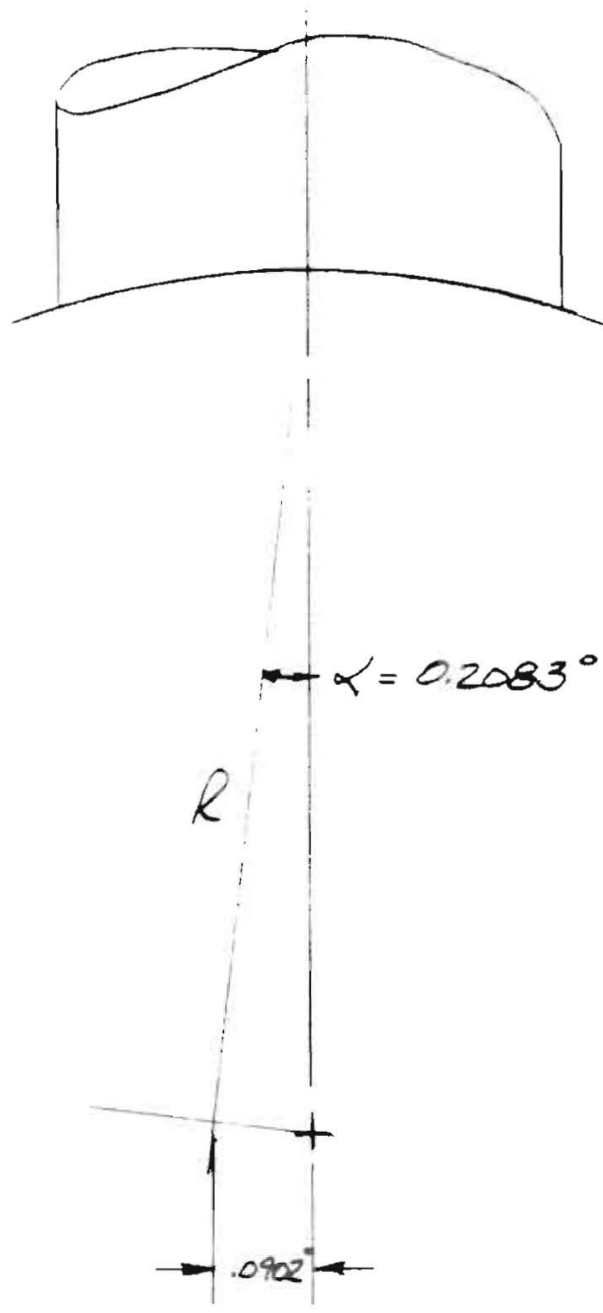

FWOR SUCI TTAT THE CENTER MOVES $0.0902^{\circ}$ ( $0.35^{\circ}$ ) WHEN ROTATED $0.2083^{\circ}\left(.147^{\circ}\right)$

\begin{tabular}{l|c}
$\sin \alpha=\frac{.0902}{R}$ & \\
$R=\frac{0.0902^{\prime \prime}}{\sin \left(.2083^{\circ}\right)}$ & $\frac{.0635}{.00257}$ \\
& $=24.814^{\prime \prime}$ \\
use $R=24 \frac{13}{16}^{\prime \prime}$ & $24.71^{\prime \prime}$ \\
Use $R=24 \frac{11}{16}$
\end{tabular}

Revisec

9/23/87

seluther 
cese 8/7/87

3

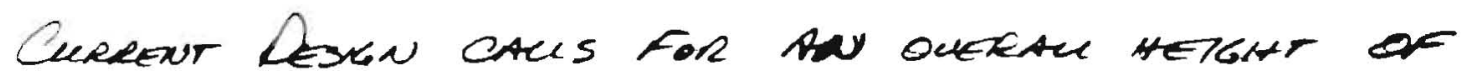

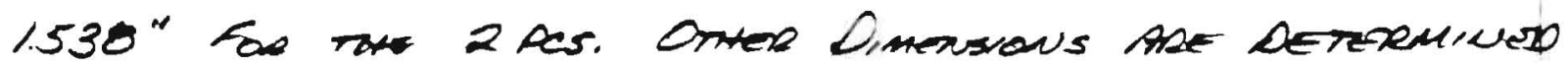

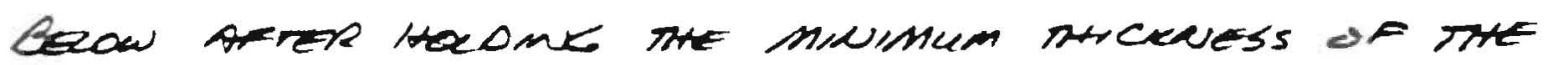

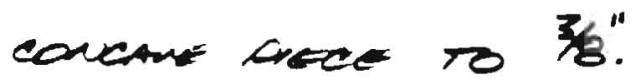
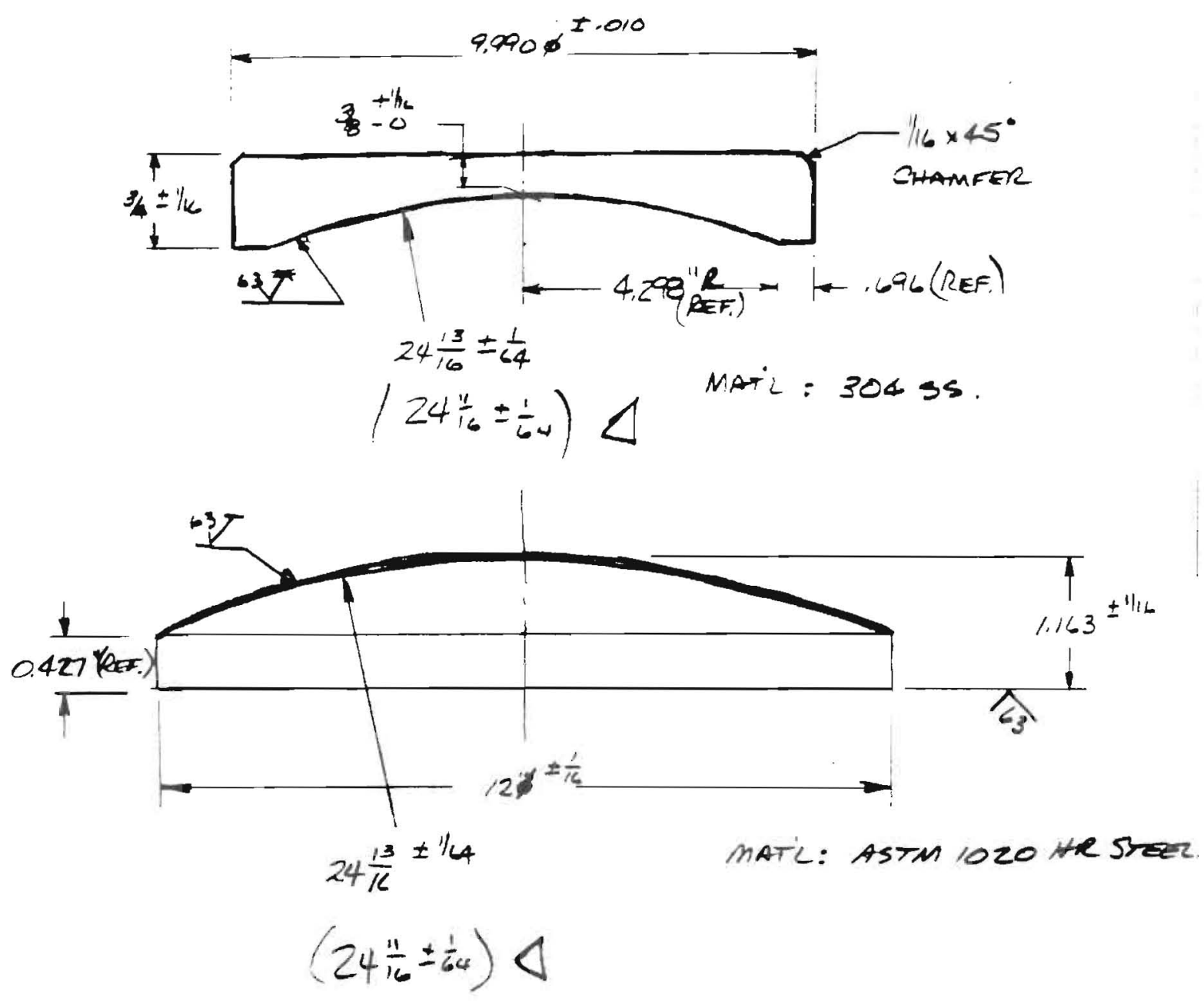
2

Apapowix A Ear Not rios

Jo, CW

$2-2\lfloor-8]$

BK. $0075=1$
DA DETECTOR

CENTRAL CALORIMETER SUPPORT LOCATED AT LAB

FOR INFORMATION ON LOAD TEST SET UP SEE DRAW IMO $3740-M 0-123703$ :

PERSON IN CHARGE 15 RICK LUTHER EXT ZZZ PAGE 781

HORIZONTAL MEASUREMENTS AT LOCATIONS SWOWM . ON pi $\pi$ HIS POX.

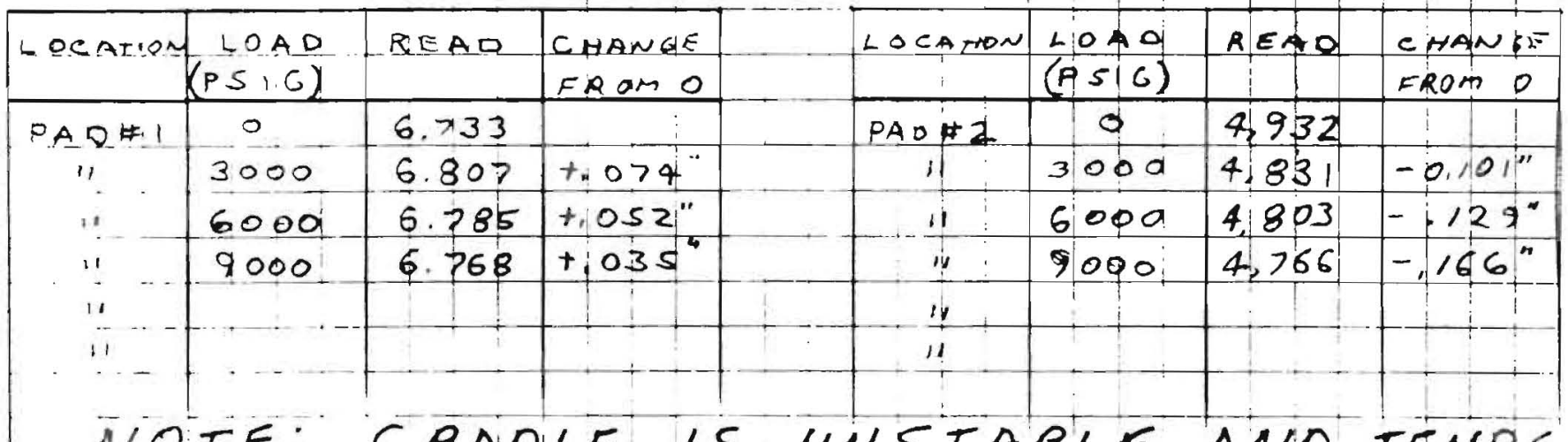

NOTE: CRADLE IS UNSTABLE AND TENDS TO MOVE DURING LOAD CHANGES.

NOTE: A POSITIVE CHANGE ON PADS 1 \& AND A NEGATIVE SHAME ON PADS 493 . DENOTES AN OVERALL MOUE IN THE SAME ORECTLON

HORIZONTAL MEASUREMENTS AT LOCATING SHOWN ON PAGE 3.TMISBOON.

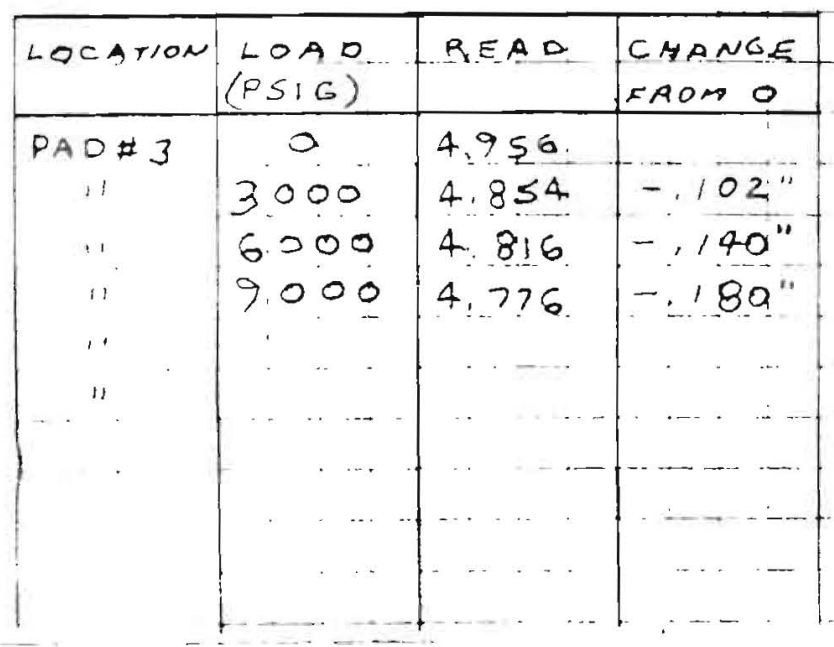

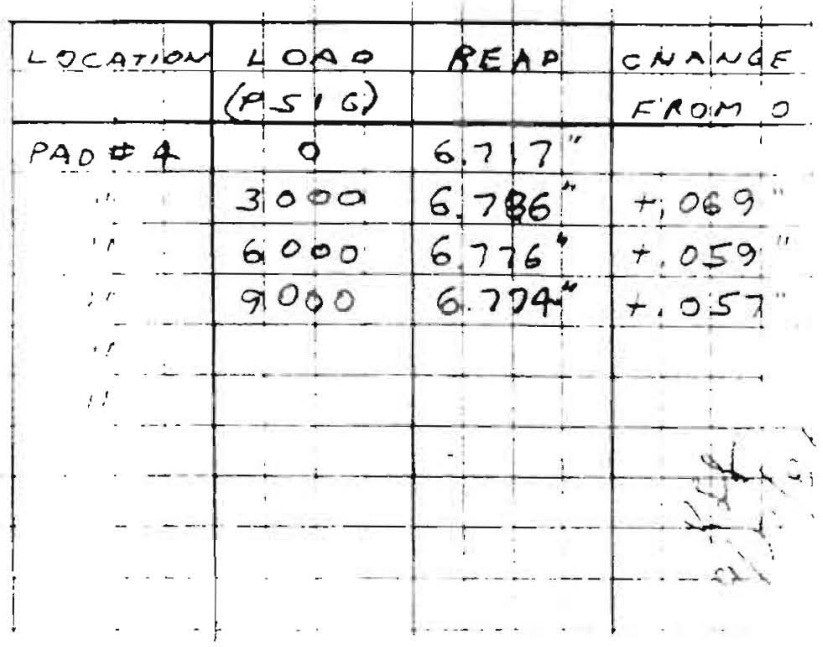


JO, $<\omega$

$9-21-87$

ER DO? $>-1$.

SUPPORT ERAOLE LOAO TEST CONJANUEO EROM P 2 TMS BK.

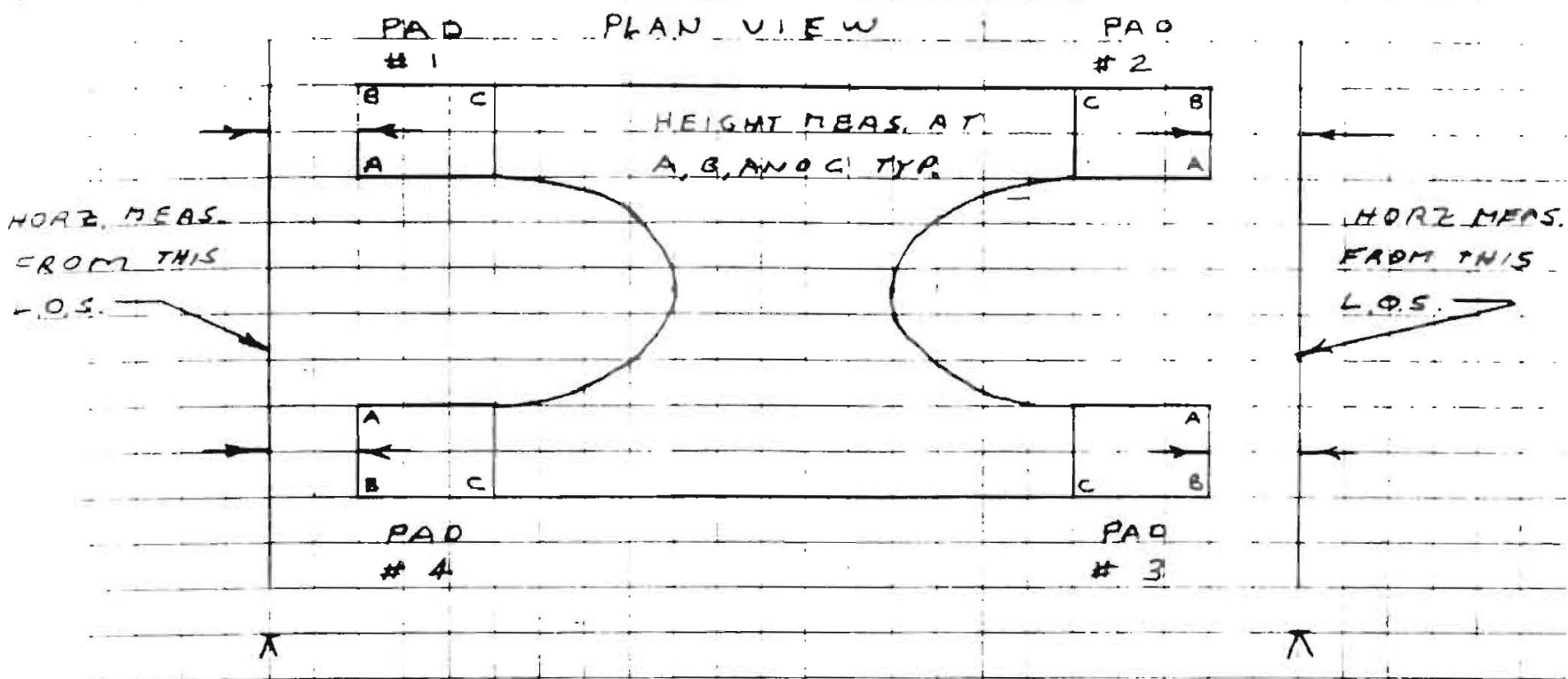

FRONT VIEW

IOOPZ MEAS HEAE. 
JD.CW . SUPPORT GAARLE LOAD TEST CONTINUAD

$9-21-8=$

FROA P 3 THAS QK.

$B K D 0>5-1$

LOCATION PSIG FBSE HE

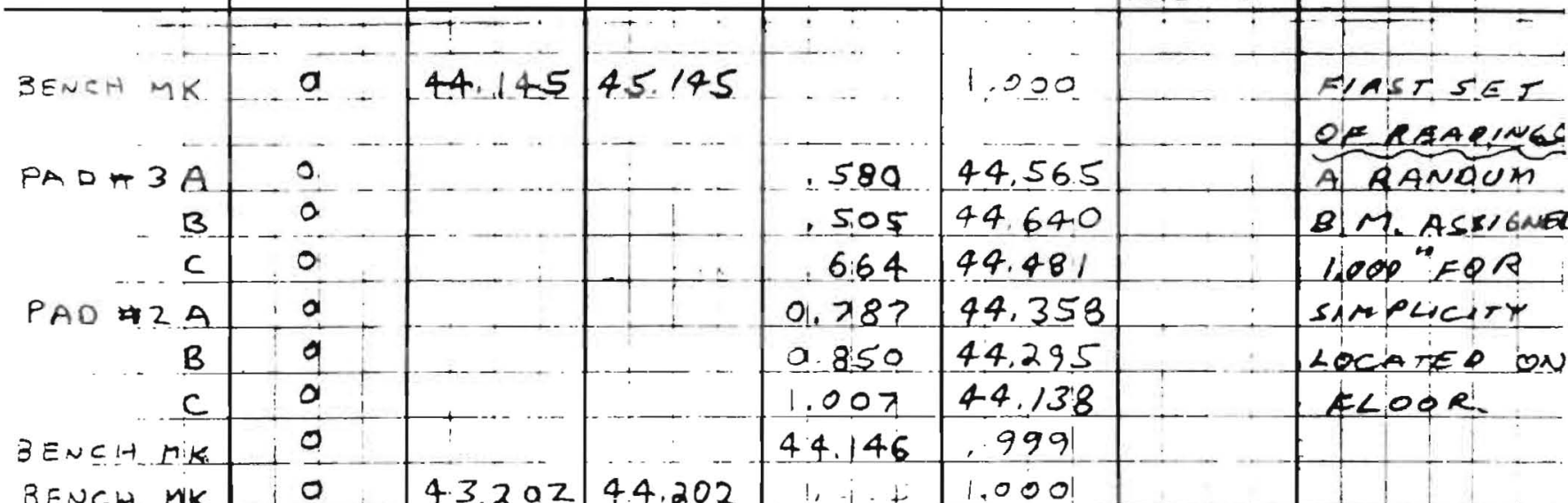

\begin{tabular}{r|c|c|} 
BENCH MK & 0 & 43202 \\
PAO IA & 0 & \\
$B$ & 0 & \\
$C$ & 0 &
\end{tabular}

44.202

$P A_{b} \# 4 A$

A $\quad 0$

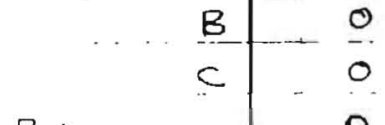

$B M$

3M

$P A D \# 1$

\begin{tabular}{l|c|c} 
& 0 & 0 \\
A. & 3000 & 43.200 \\
\hline B & 3000 & \\
\hline & 3000 & \\
\hline & 3000 &
\end{tabular}

$P A D \# 4 A 3009$

B 3000

$B \dot{M}$

C 3000

$3 m$

\begin{tabular}{r|r} 
PAD $3 A$ & 3000 \\
$B$ & 3000 \\
$C$ & 3000 \\
PAO 2 A & 3000 \\
$B$ & 3000 \\
$B$ & 3000 \\
$B M$ & 3000
\end{tabular} 3000

B.M

PAD $3 A$ - 6000

\begin{tabular}{c|cc}
$B$ & 6000 \\
$C$ & 6000 \\
\hline
\end{tabular}

PAO $2 A$ A 6000

B 6000

44,146

44.146

$45 \cdot 146$

$0: 8: 17$

$0,738^{\circ}$

$0.827^{\circ}$

$11101^{\prime \prime}$

1.175

1.269

44.146

$45.146^{n}$

0.910

0.828

0.898

1.187

$.25 ?$

1.326
1.0001

42,928

$42,864^{n}$

$43.012^{n}$

$43.085^{n}$

43,141

$43,312^{\prime \prime}$

$1,000^{n}$

43.072

$+.144^{\circ}$

$42.992^{\circ}+28$

43.107

$+.0955^{\circ}$

$+186^{\circ}$

$43.353+.212$
$43,490+.178$

1,002

$1.000^{\circ}$

$44,329^{\prime \prime}-.236$

$44,408^{\circ}-232$

$44.319^{n}-62$

$44.045^{\circ}$

$43.971^{\circ}$

$-.3 / 3$

$43.921-22$

$43,877^{n}-.26$

1,000

$1.000^{\circ}$

$44.230^{\circ}$

$44,318^{\circ}$

$-.379$

$-\quad 323$

$44.248^{\prime \prime}$

43.959

43,839

43.325 $\pi 0$

t-2

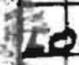

每
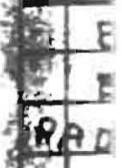

龺

运

QAD

.

$+$

PAD

等.

plo

13

C.HANGE DSIG

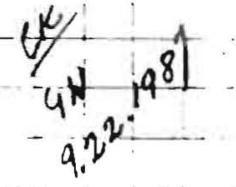

CHANGE:PSIG

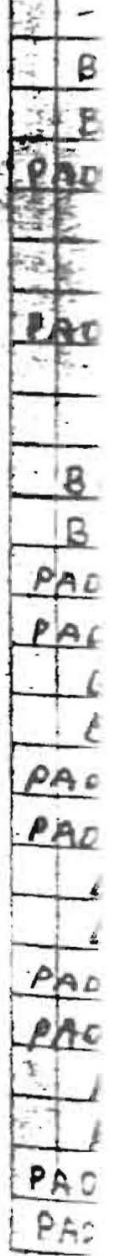


$\tau D, C \omega$

9-2, $1-87$

$8 \times 00>5-1$
SUPPORT CRAPLEI LOAD TEST COMYNYES From R.4 THIS BK.
IE.

sanenger Naun

ASEIGME:

FQR

ICATH

$T \in 0$ on

QR.

\begin{tabular}{|c|c|c|c|c|c|c|c|}
\hline LEA MIOU & $0.5 / 6$ & B.S. & it I & F.S. & $H E, G A T$ & $\begin{array}{l}\text { CHANOE } \\
\text { FAOM O }\end{array}$ & PFMARR \\
\hline$B M$ & 6000 & & & 44.145 & $1,001^{\circ}$ & & \\
\hline BM & 6000 & 43.201 & 44,201 & $\ldots=$ & 1.000 & & \\
\hline$P A D \perp \mid A$ & 6000 & $-50-2$ & $-\infty$. & 1,158 & 43.043 & $\pm .115^{\circ}$ & . \\
\hline & 6000 & & & $1.232^{\circ}$ & 42.969 & $+105^{\circ}$ & $\cdot$ \\
\hline & 6000 & & & $1109^{\circ}$ & 43.092 & $+.080^{\circ}$ & . \\
\hline PAOE $4 A$ & 6000 & & & $0.953^{\circ}$ & $43,248^{\prime}$ & $+163^{\prime \prime}$ & . \\
\hline & 6000 & & & $0.869^{\circ}$ & $43.332^{\prime \prime}$ & $+.191^{\circ}$ & 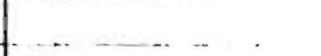 \\
\hline$c$ & 6000 & & & $0.723^{\prime \prime}$ & $43,478^{\prime \prime}$ & +.166 & \\
\hline $\mathrm{Bn}$ & 6000 & 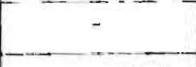 & & $43.203^{4}$ & $.998^{\circ}$ & & \\
\hline$B M$ & 9900 & 44.146 & 45,146 & $1:$ & 1.000 & & CHANCE PSIG \\
\hline PAD $3 A$ & 9000 & & & 1. 305 & 43.841 & -.517 & TO MAXMUM \\
\hline$B$ & 9000 & & & $1,3 \geq 1$ & $43,7>5$ & -.520 & $\angle O A O$ \\
\hline$<$ & 9000 & & & 1415 & $43,731^{n}$ & $-407^{\circ}$ & \\
\hline PAD $A A$ & 9000 & & & 1,006 & 44,140 & $-.425^{\prime \prime}$ & \\
\hline$B$ & 9000 & & & 0.926 & 44.220 & $-.420^{\prime \prime}$ & \\
\hline$c$ & 9000 & & & 0.902 & 44,174 & $-307^{\circ}$ & \\
\hline$B M$ & 9000 & & & 44.149 & $992^{\prime \prime}$ & & \\
\hline$B M$ & 9000 & 43,203 & 44.203 & & 1,000 & & \\
\hline PAOHIA & 9000 & & & 1,184 & $43, D 19$ & $+091^{\prime \prime}$ & \\
\hline & 9000 & & & 1.256 & 42,947 & $+0.83^{\circ}$ & \\
\hline & 0000 & & & $1.129^{\circ}$ & $4,3,074$ & $+062^{\prime \prime}$ & \\
\hline PADE $4 A$ & 9000 & & & 0.9160 & 43.243 & $+158^{\prime \prime}$ & $\vdots$ \\
\hline .... B & 9000 & & & $0.8>0$ & 43.333 & $+192^{\circ}$ & \\
\hline$c$ & 9000 & & & $0,7 \geq 2$ & 43,481 & $+169^{\prime \prime}$ & \\
\hline$B M$ & 9000 & & & 43.201 & 1.0011 & : : & \begin{tabular}{l|l|l}
$\vdots$ & & \\
\end{tabular} \\
\hline$B M:$ & 0 & 43.202 & 44,202 & $\vdots \vdots \vdots$ & 1.000 & $1:$ & RETUEN TO \\
\hline$\rho_{A O}=1 B$ & $a$ & & & $1.313^{\prime \prime}$ & 42.889 & +.025 & 0 AS/G \\
\hline PAOH 48 & $\therefore a$ & & & $1.014^{\prime \prime}$ & 43.188 &,+ 047 & secon a ser \\
\hline$B N$ & $\therefore$ & & & $4,3.203$ & .999 & & OF REARINOS \\
\hline$B M$ & $a$ & 44,147 & 45,147 & & 1.000 & & \\
\hline PAOF $2 B$ & 0 & & & $0.9: 54$ & 44.193 &.,$- 102 "$ & \\
\hline PAO $3 B$ & 0 & & & $0.592^{\circ}$ & 44.555 & $-085^{\circ}$ & \\
\hline$B M$ & 0 & & & 44.146 & 1,001 & 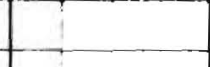 & $5 ! \div \div \ldots$ \\
\hline$B M$ & 6000 & $4: 4.146$ & 4,$5 ; 146$ & & 1.000 & & CMANUEPSIG \\
\hline PAD $2 B$ & 6,000 & & & $1.320^{\circ}$ & 43,826 & -.367 & CHANCES \\
\hline PAOE $3 B$ & 6000 & & & $0.8169^{\prime \prime}$ & 44,277 & $-\quad 228$ & RELIATILS TO \\
\hline$B M$ & 6000 & & & 44.146 & $1.000^{\prime \prime}$ & & SKECP O SET \\
\hline$B M$ & 6000 & 43.202 & 49.202 & 1 & $1,000^{\circ 1}$ & + & dF 0 psik \\
\hline PAOHIB & 6000 & $\vdots \quad \ldots$ & $\ldots \vdots \vdots$ & $1: 211:^{\prime \prime}$ & $42.991^{\prime \prime}$ & +102 & REAOINES I \\
\hline PAO $A B$ & 6000 & & 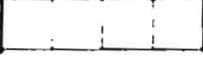 & 0.841 & $43.361^{1}$ & +.173 & \\
\hline
\end{tabular}


6

SD, CW

$9,-2)-8 ?$

BK DO $>5-1$

\begin{tabular}{|c|c|c|c|c|c|c|c|c|c|}
\hline \multirow[t]{2}{*}{ STATION } & \multirow[t]{2}{*}{$P S 1 G$} & & \multirow[t]{2}{*}{5} & $H I$ & AS & HENGNI & CAANLE & \multicolumn{2}{|c|}{ REMARKS } \\
\hline & & & & & & & Fioris o & & \\
\hline$B M$ & 6000 & & 1 & & 43.204 & .998 & $1: 1$ & & \\
\hline$B M$ & 9000 & \multicolumn{2}{|c|}{43.204} & 44.204 & & 1.000 & & & \\
\hline$P A D+\perp B$ & 9000 & & & 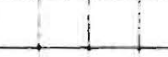 & 1.248 & 42.756 & +067 & \multicolumn{2}{|c|}{ crondetra } \\
\hline PAO $\triangle B$ & 9000 & & & & 0.864 & 43,340 & +.152 & & \\
\hline$B M$ & 9000 & & & & 43.202 & 1.002 & & & \\
\hline $\mathrm{BM}$ & 9000 & \multicolumn{2}{|c|}{$44: 149$} & $4 \sin 149^{\prime \prime}$ & & 1.000 & 1 & & \\
\hline PAD $\# 2 B$ & 9000 & & & & $1.392^{\circ}$ & $4,3.757$ & $-.436^{4}$ & & \\
\hline PAD 38 & 9000 & & & & $0.9134^{n}$ & \multirow{2}{*}{$\frac{44.215}{1.002}$} & $-340^{4}$ & & \\
\hline$B M$ & 9000 & & & & 44.147 & & & & \\
\hline & $i i$ & & & & & & & & \\
\hline & 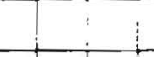 & & & & & & & & \\
\hline & & & & & & & & & \\
\hline & 1 & & & & & & & & \\
\hline$\therefore$ & $\vdots$ & & & ' & & & & 1 & \\
\hline & 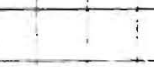 & & & & & & & & \\
\hline & & & & & & & 7 & & \\
\hline & & & & & 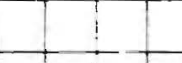 & & $\vdots$ & & 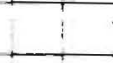 \\
\hline & & & $\therefore$ & & & & & & \\
\hline & U & & & & 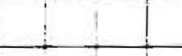 & & & 1 & \\
\hline & & 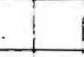 & & & & & & & \\
\hline & & 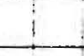 & & & & & & & \\
\hline
\end{tabular}

support daAola boAd rasir continueo

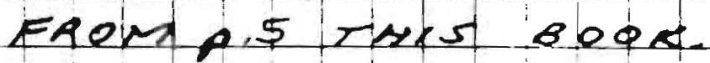

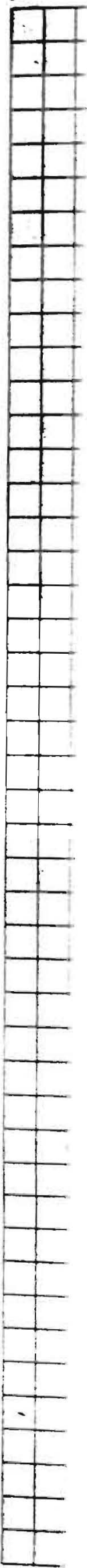



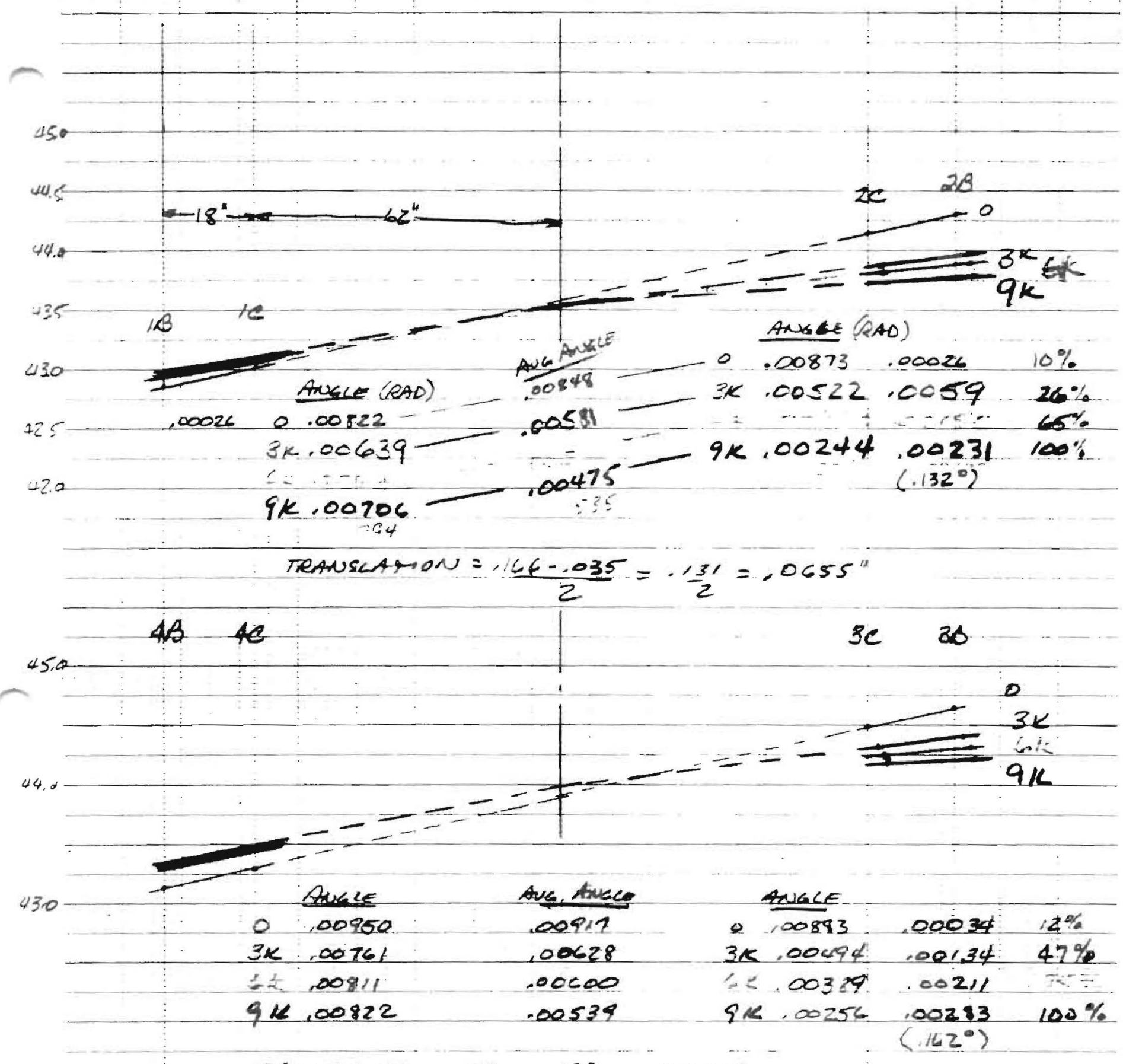

rears $=\frac{180-.057}{2}=\frac{123}{2}=.0615$

Aud Eor $=.00231+.0085$

$=.147^{\circ}$

Huc taAns $=\frac{0.055+.0615}{2}$ 


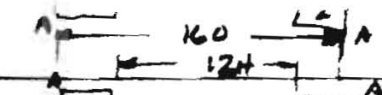

PRD $1 B$

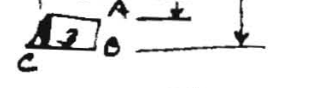

LAC NF

0 o 42864

$3<0^{*} 42992$, $=$ ?

9

$6 \times 0^{5} 42969=$

a<. 42.947

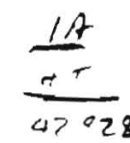

Ie
43.012 .095
43.107 .015
$43.092 .0:==$
43.074

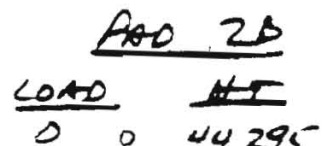

43.092

43.04332

$4 \geq 019^{-224}$

o 42.889

$611 \quad 42.991$

ak 42.956

$\begin{array}{lll}0 & 0 & 495 \\ 3 k & .01 & 43.975^{-32}\end{array}$

6k.12 43889 os

9k. $1^{66} 43775$

0

$6 / 2$

4.193

$9 k$

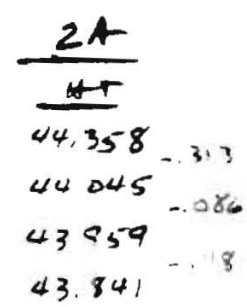

2e

44.38 43.977

43.820

43.791

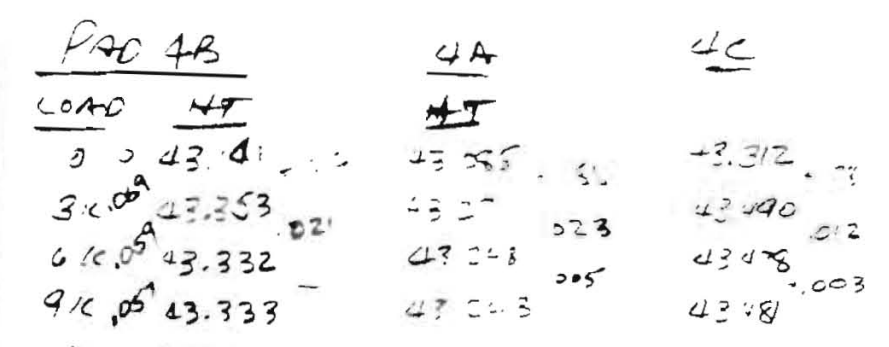

$0 \quad 43.88$

6/k 43.26: 02

$9 / C_{4}+3.340^{\circ}$

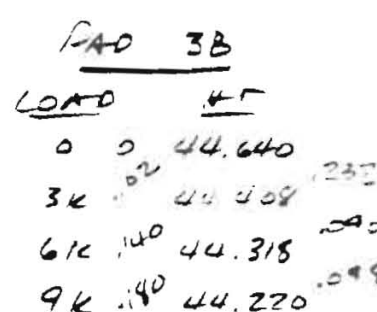

$3 A$

30

it

44.565

a) ?29

44.236

$44,140.094$

0 u4. 555 .

bic 14.27

ile $44.215^{=}=$

TeAnsethons

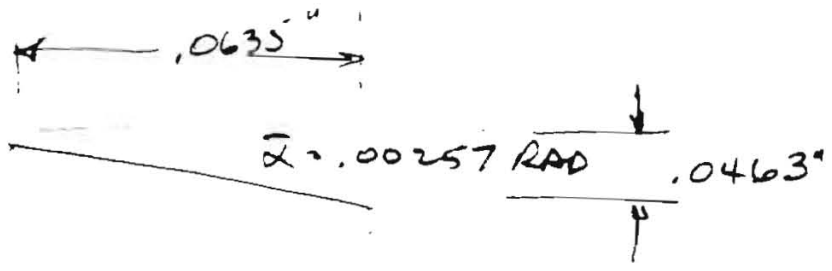

$$
r=\frac{0.0635}{.00257}=24.71^{n}
$$

